\title{
A Memory-Based Hysteresis Model in Piezoelectric Actuators
}

\author{
Guilin Zhang, ${ }^{1}$ Chengjin Zhang, ${ }^{1}$ and Jason Gu$^{1,2}$ \\ ${ }^{1}$ School of Control Science and Engineering, Shandong University, Jinan 250061, Shandong, China \\ ${ }^{2}$ Electrical and Computer Engineering, Dalhousie University, Halifax, NS, Canada
}

Correspondence should be addressed to Chengjin Zhang, cjzhang@sdu.edu.cn

Received 12 January 2012; Revised 25 March 2012; Accepted 5 April 2012

Academic Editor: Pak Kin Wong

Copyright () 2012 Guilin Zhang et al. This is an open access article distributed under the Creative Commons Attribution License, which permits unrestricted use, distribution, and reproduction in any medium, provided the original work is properly cited.

\begin{abstract}
A mathematical memory-based model is proposed to capture the hysteresis behavior in piezoelectric actuators. It is observed that the ascending (descending) hysteresis curves are alike and converge to one point without memory saturation. Therefore, two, dominant curves are determined and expressed as continuous functions, and the other hysteresis curves are modeled using two dominant curves through nonlinear transforming of coordinate axis. In the event of memory saturation, a new converging point is used to compensate the model prediction error. The experimental study has been carried out and our proposed model prediction method is compared with PI model and the linear model. It shows that the proposed model prediction method is better than other two methods.
\end{abstract}

\section{Introduction}

Piezoelectric ceramics are widely used as actuators in nano-/ micropositioning mechatronic systems due to their fast frequency response, nanometer scale resolution, and high stiffness. Since the materials of piezoelectric actuators are ferroelectric, nonlinear hysteresis behavior is commonly observed in such actuators in response to an applied electric field. Thus, it is very challenging to design high-performance servo controller for nano-/micropositioning mechatronic systems [1].

In the past few years, many studies on the compensation for the positioning of the piezoelectric actuators have been reported. One simple useful method is the model-based feedforward control scheme. This method cascades an inverse hysteresis model in series with an actuator plant to cancel out the effect of nonlinearity and achieve a relatively linear response. The more precision the inverse hysteresis model is, the better the control scheme tracking performance is. Consequently, modeling hysteresis is the first step to track control of piezoelectric actuators.

Numerous research works have been done to model the hysteresis nonlinearity of piezoelectric actuators. The most well-known hysteresis model is the Preisach model [2-11]. It is the broadly used approach in modeling and compensation of hysteresis in piezoelectric actuators. However, Preisach model does not have an analytical inverse. Ge and Jouaneh improved classical Preisach model that can predict the hysteresis response of a piezoelectric actuator driven by a periodic sinusoidal or triangular input signal [5] and developed a computer-based tracking control approach for piezoelectric actuators based on linearizing the hysteresis nonlinearity [6]. Tan et al. formulated and proposed the value inversion algorithm using a class of discretized Preisach operators [9]. They proposed an adaptive identification of hysteresis in smart materials and developed an adaptive inverse control scheme to update the weight of Preisach operators [10].

Another popular hysteresis model is Prandtl-Ishlinskii (PI) model, which is a subclass of the Preisach model. The advantage of PI model is that its inverse is also a PI type with different threshold and weighting values. Kuhnen developed an inverse scheme based on the inverse scheme of PI model $[12,13]$. However, the PI operator in PI model has a symmetry property around the center of the loop, while hysteresis response of a piezoelectric actuator is not symmetric in practice. Bashash and Jalili investigated the nature of hysteresis in piezoelectric materials and discussed a modified PI model that describes the asymmetric and residual displacement properties simultaneously [14]. Jiang et al. proposed another modified PI model based on two asymmetric operators to model the asymmetric property of hysteresis [15]. The other disadvantage of PI model is 
that it cannot compensate for the saturated hysteresis. Al Janaideh Mohammad et al. proposed a generic PI model based on a generalized play operator with different loading and unloading envelop functions in order to characterize asymmetric and saturated hysteresis nonlinearities [16]. They also proposed the modified generalized PI model that has the exact analytical inverse [17].

$\mathrm{Xu}$ and Wong applied least squares support vector machine (LS-SVM) to capture the rate-independent and rate-dependent hysteresis nonlinearities $[18,19]$. Moreover, a set of models are proposed to describe the hysteresis properties including targeting turning points, curve alignment and wiping-out property. Tzen et al. applied an exponential curve to fit the hysteresis path when the piezoelectric actuator operated away from the saturation [20]. Sun et al. proposed a hysteresis model based on similarities of the hysteresis curves and the turning points [21]. Bashash and Jalili disclosed the memory-dominant nature of hysteresis in piezoelectric materials and developed a memory-based mathematical hysteresis model [22-24]. They adopted the proposed model in an inverse model-based control scheme for feedforward compensation of hysteresis nonlinearity [22].

In this paper, we adopt the mathematical transformation to describe the hysteresis curve functions. The ascending and descending loading curves can be determined and expressed as two continuous functions. Then the rest of hysteresis curves adopt their shape from the dominant curves. In addition, a new converging is updated to compensate the model prediction error in the event of memory saturation. It is demonstrated that the proposed model prediction is noticeably improved compared with the model without memory operator.

The remainder of this paper is organized as follows. First, a mathematical memory-based hysteresis model in piezoelectric actuators is proposed in Section 2. Moreover, the approximate congruency property of the proposed model is validated in this section. Then the experimental setup and the proposed model performances are given in Section 3. In Section 4, a new converging point is updated to address the memory saturation. Finally, some concluding remarks are provided in Section 5.

\section{Hysteresis Model}

Hysteresis trajectory starts moving on the ascending loading curve as depicted in Figure 1. This curve can be approximated by a monotonically increasing continuous function. As shown in Figure 1, the ascending curves are alike and converge to the upper targeting point. When the direction of the input changes, the trajectory breaks its path and moves downward on the descending loading curve that can be approximated by another monotonically increasing continuous function. All the descending curves are also alike and converge to the lower converging point. The ascending and descending dominant curves can be expressed as two monotonically continuous functions, $f_{r a}(v)$ and $f_{r d}(v)$, respectively. In addition, the rest of hysteresis curves can adopt their shape from these dominant curves.

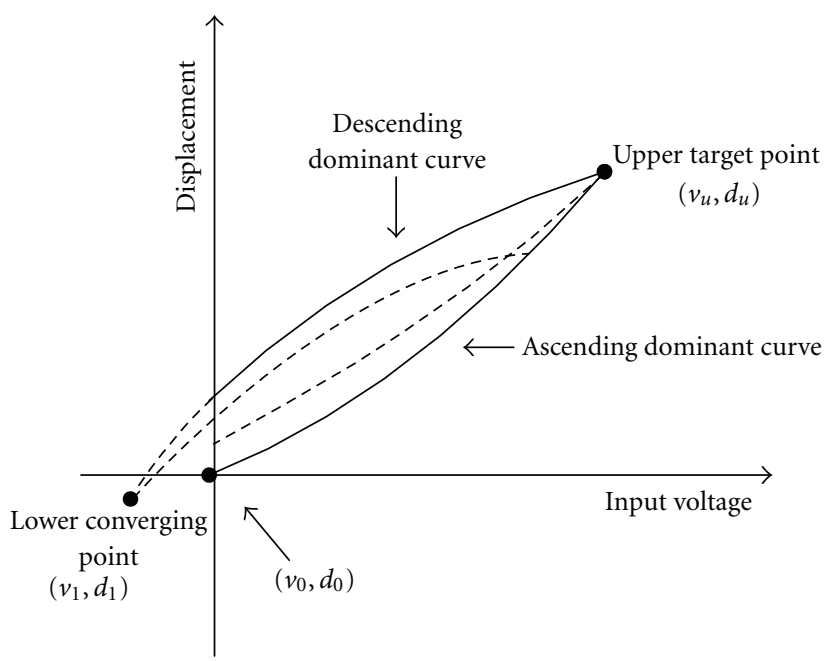

FIGURE 1: Ascending and descending dominant curves.

For any ascending trajectory starting from point $\left(v_{1}, d_{1}\right)$ and $\left(v_{2}, d_{2}\right)$, the following linear function is obtained:

$$
d_{a}(v)=k_{a} f_{r a}(v)+b_{a},
$$

where $d_{a}(v)$ represents the ascending hysteresis trajectory between points $\left(v_{1}, d_{1}\right)$ and $\left(v_{2}, d_{2}\right)$, when the input voltage $v$ varies between $v_{1}$ and $v_{2}, k_{a}$ and $b_{a}$ are given by

$$
k_{a}=\frac{d_{u}-d_{1}}{f_{r a}\left(v_{u}\right)-f_{r a}\left(v_{0}\right)}, \quad b_{a}=d_{1}-k * f_{r a}\left(v_{0}\right),
$$

where $v_{0}$ and $v_{u}$ are the initial voltage and the upper converging voltage of the dominant curve depicted in Figure 1.

Replacing $k_{a}$ and $b_{a}$ from (2) into (1) yields

$$
\begin{aligned}
d_{a}\left(v, v_{1}, d_{1}, v_{2}, d_{2}\right) & \\
= & d_{1}+\frac{d_{u}-d_{1}}{f_{r a}\left(v_{u}\right)-f_{r a}\left(v_{0}\right)} \\
& \times\left(f_{r a}(v)-f_{r a}\left(v_{0}\right)\right) .
\end{aligned}
$$

Once the initial voltage $v_{1}$ and displacement $d_{1}$ are decided, the hysteresis curves are obtained by using the dominant curve. However, when the initial voltage $v_{1}$ substitute $v$ in (3),

$$
\begin{aligned}
& d_{a}\left(v_{1}\right) \\
& \quad=d_{1}+\frac{d_{u}-d_{1}}{f_{r a}\left(v_{u}\right)-f_{r a}\left(v_{0}\right)}\left(f_{r a}\left(v_{1}\right)-f_{r a}\left(v_{0}\right)\right) \neq d_{1} .
\end{aligned}
$$

Then, the ascending curve function is modified as

$$
\begin{aligned}
& d_{a}(v) \\
& \quad=d_{1}+\frac{d_{u}-d_{1}}{f_{r a}\left(v_{u}\right)-f_{r a}\left(v_{0}\right)}\left(f_{r a}\left(m v+(1-m) v_{u}\right)-f_{r a}\left(v_{0}\right)\right),
\end{aligned}
$$


where $m=\left(v_{u}-v_{0}\right) /\left(v_{u}-v_{1}\right)$ and when $v=v_{1}, d_{a}\left(v_{1}\right)=$ $d_{1}$ and when $v=v_{u}, d_{a}\left(v_{u}\right)=d_{u}$. It demonstrates that the model can start from the initial displacement and reach the upper converging point.

Similarly, when the input voltage $v$ varies between $v_{1}$ and $v_{2}$, the descending curve function is given by

$$
d_{d}(v)=k_{d} f_{r d}(n v+(1-n))+b_{d},
$$

where $d_{d}(v)$ represents the descending hysteresis trajectory between points $\left(v_{1}, d_{1}\right)$ and $\left(v_{2}, d_{2}\right), k_{d}$ and $b_{d}$ are the same as $k_{a}$ and $b_{a}$ and given by

$$
\begin{gathered}
k_{d}=\frac{d_{l}-d_{1}}{f_{r d}\left(v_{l}\right)-f_{r d}\left(v_{u}\right)}, \\
b_{d}=d_{1}-k_{d} * f_{r d}\left(v_{u}\right), \quad n=\frac{v_{l}-v_{u}}{v_{l}-v_{1}},
\end{gathered}
$$

where $\left(v_{u}, d_{u}\right)$ is the upper converging point and $v_{u}$ is also the initial voltage of the descending dominant curves, $\left(v_{l}, d_{l}\right)$ is the lower converging point and can be identified. Then (6) can be transformed into

$$
\begin{aligned}
& d_{d}\left(v, v_{1}, d_{1}, v_{2}, d_{2}\right) \\
& \quad=d_{1}+\frac{d_{l}-d_{1}}{f_{r d}\left(v_{l}\right)-f_{r d}\left(v_{u}\right)}\left(f_{r d}\left(n v+(1-n) v_{l}\right)-f_{r d}\left(v_{u}\right)\right) .
\end{aligned}
$$

The congruency property states that any two minor hysteresis loops are identical and have the same shape if they are generated by back-and-forth variations of the input between two identical extrema. Here, we consider the case of two points $\left(v_{x}, d_{x 1}\right)$ and $\left(v_{x}, d_{x 2}\right)\left(d_{x 1} \neq d_{x 2}\right)$. When the input voltage reaches $v_{y}$, the displacement $d_{y 1}$ and $d_{y 2}$ are obtained by using (5).

$d_{y 1}$

$$
=d_{x 1}+\frac{d_{u}-d_{x 1}}{f_{r a}\left(v_{u}\right)-f_{r a}\left(v_{0}\right)}\left(f_{r a}\left(m v+(1-m) v_{l}\right)-f_{r a}\left(v_{0}\right)\right),
$$

$d_{y 2}$

$$
=d_{x 2}+\frac{d_{u}-d_{x 2}}{f_{r a}\left(v_{u}\right)-f_{r a}\left(v_{0}\right)}\left(f_{r a}\left(m v+(1-m) v_{l}\right)-f_{r a}\left(v_{0}\right)\right) .
$$

Subtracting (9) from (10) yields

$$
\begin{aligned}
d_{y 2}- & d_{y 1} \\
= & d_{x 2}+\frac{d_{u}-d_{x 2}}{f_{r a}\left(v_{u}\right)-f_{r a}\left(v_{0}\right)} \\
& \times\left(f_{r a}\left(m v_{y}+(1-m) v_{u}\right)-f_{r a}\left(v_{0}\right)\right) \\
& -d_{x 1}+\frac{d_{u}-d_{x 1}}{f_{r a}\left(v_{u}\right)-f_{r a}\left(v_{0}\right)} \\
& \times\left(f_{r a}\left(m v_{y}+(1-m) v_{u}\right)-f_{r a}\left(v_{0}\right)\right)
\end{aligned}
$$

$$
\begin{aligned}
& =\left(d_{x 2}-d_{x 1}\right)\left(1+\frac{f_{r a}\left(m v_{y}+(1-m) v_{u}\right)-f_{r a}\left(v_{0}\right)}{f_{r a}\left(v_{u}\right)-f_{r a}\left(v_{0}\right)}\right) \\
& =\left(d_{x 2}-d_{x 1}\right)(1+\sigma) .
\end{aligned}
$$

When $v_{y} \ll v_{u}, \sigma \approx 0$, then $d_{y 2}-d_{y 1} \approx\left(d_{x 2}-d_{x 1}\right)$. That is to say, the congruency property of the proposed model can be approximately satisfied only under the condition that the input voltage is far away from the upper converging point.

The implementation of the model needs to identify the ascending and descending dominant curves. Some structures have been proposed for these curves, including secondorder polynomials, third-order polynomials, and exponential functions. Here, two-third-order polynomials are used for the approximation of the ascending and descending dominant curves. The polynomials are expressed as

$$
\begin{aligned}
& f_{r a}(v)=\sum_{i=0}^{3} a_{i} v^{3-i}=a_{0} v^{3}+a_{1} v^{2}+a_{2} v+a_{3}, \\
& f_{r d}(v)=\sum_{i=0}^{3} d_{i} v^{3-i}=d_{0} v^{3}+d_{1} v^{2}+d_{2} v+d_{3},
\end{aligned}
$$

where $a_{i}$ and $d_{i}$ are the dominant curve coefficients that can be identified by utilizing the least square identification method.

The proposed model has several advantages compared to the Preisach model. Firstly, it only needs eight parameters compared with the Preisach model which needs a large number of parameters. Secondly, the numerical inversion of the proposed model can be easily obtained in real time based on the mathematical formulation.

\section{Experimental Setup and Model Verification}

To investigate the effectiveness of the proposed piezoelectric actuator hysteresis model, a set of experiment on a PST150/7/40VS12 PZT-driven is demonstrated using the PC-based control system. Figure 2(a) shows the experimental block diagram. The setup includes a personal computer, a power controller, and a piezoelectric actuator, which provides maximum $40 \mu \mathrm{m}$ displacement and an integrated high-resolution strain gauge position (SGS) sensor. The host computer generates the control codes, which are written in Visual $\mathrm{C}++$ and run through the EPP (Enhanced Parallel Port). The digital signal is converted by 16 -bit $\mathrm{D} / \mathrm{A}$ converter and amplified by a high-voltage amplifier (PVC-150S1). The actual actuator output displacements are measured by a SGS sensor and converted to a digital signal by a 16-bit $\mathrm{A} / \mathrm{D}$ converter. Then the displacement data are sent to the host computer. The experimental setup (without PC) is shown in Figure 2(b).

The dominant curves should be obtained first. A firstorder reversal signal is applied to excite the piezoelectric actuator, which is shown in Figure 3(a). The proposed model is applied to predict the output displacement. Figure 3(b) 


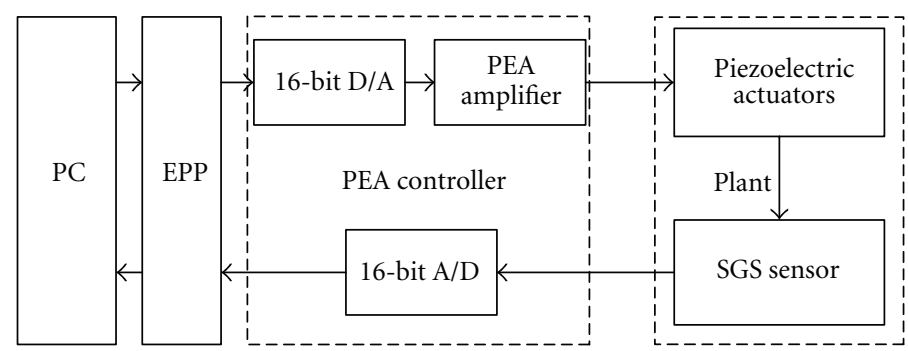

(a)

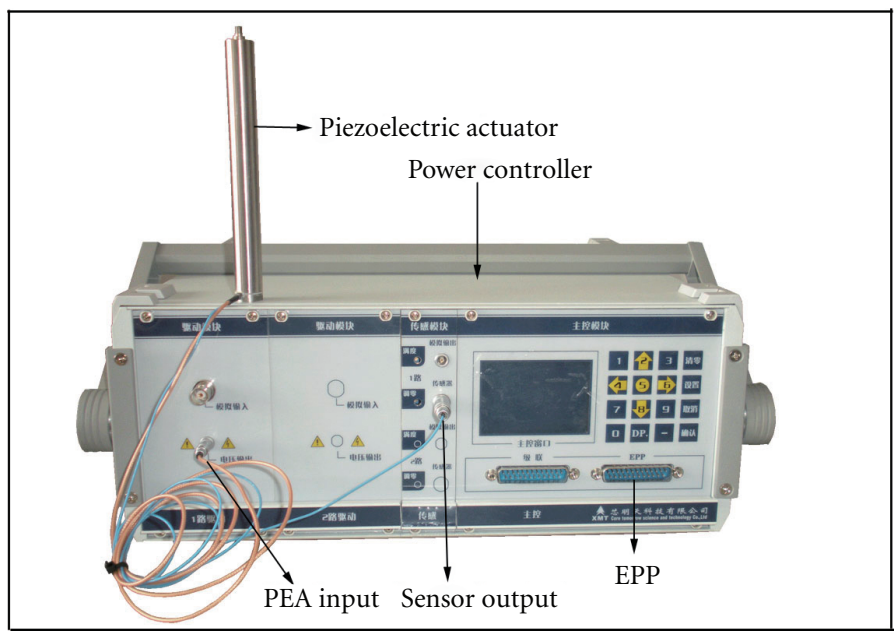

(b)

Figure 2: (a) The experimental block diagram. (b) the experimental setup.

depicts the experimental displacement and the proposed model prediction. The maximum and root-mean-square (rms) error percentages of the proposed model are $0.96 \%$ and $0.49 \%$, respectively.

To demonstrate the effectiveness of the proposed model, the linear model and PI models are considered for comparison. Both the linear and PI model are simulated based on the same input given in Figure 3(a). The responses of these models are shown in Figures 3(c) and 3(d), respectively. The maximum and rms error percentages are $4.96 \%$ and $4.10 \%$ for linear model, and $1.32 \%$ and $0.52 \%$ for PI model, respectively. The modeling performance of the proposed model has been improved compared with the linear model and PI model.

\section{Saturated Memory Model}

The upper converging point is not a fixed point. In this section, the memory saturation is considered. The point 2 is a converging point as shown in Figure 4. Path 1-2 is the dominant ascending curve. When the input signal overtakes point 2 , the new hysteresis trajectory will follow the path 2-5 that is the extended path 1-2. Meanwhile, point 5 becomes the new upper converging point after the input voltage reaches point 5 . Consequently, when the input signal exceeds a converging point, the new hysteresis trajectory will converge to a new point.
We consider the case that the $i_{\text {th }}$ ascending trajectory starts from point $\left(v_{i 1}, d_{i 1}\right)$ and $\left(v_{u 1}, d_{u 1}\right), v_{u 1}>v_{u}$. Because the converging point has varied in the next ascending curves, then the next ascending curves in (5) can be updated to (13) as follows:

$$
\begin{aligned}
d_{a}(v)= & d_{1}+\frac{d_{u 1}-d_{1}}{f_{r a}\left(v_{u 1}\right)-f_{r a}\left(v_{0}\right)} \\
& \times\left(f_{r a}\left(m v+(1-m) v_{u 1}\right)-f_{r a}\left(v_{0}\right)\right) .
\end{aligned}
$$

Different from the ascending curves, the descending curves converge to a fixed point $\left(v_{l}, d_{l}\right)$. Thus, the descending curve functions keep unchanged in the event of memory saturation.

To demonstrate the effectiveness of the saturated memory model, a simulation study is carried out. The input profile shown in Figure 5(a) is applied to excite the piezoelectric actuator. The experimental and the model responses without memory operator are demonstrated in Figure 5(b). As seen from Figure 5(b), the proposed model response diverges from the correct trajectory when it hits the dominant maximum input voltage. Then the saturated memory model response is given in Figure 5(c), which demonstrates the performance enhancement of the saturated memory model. 

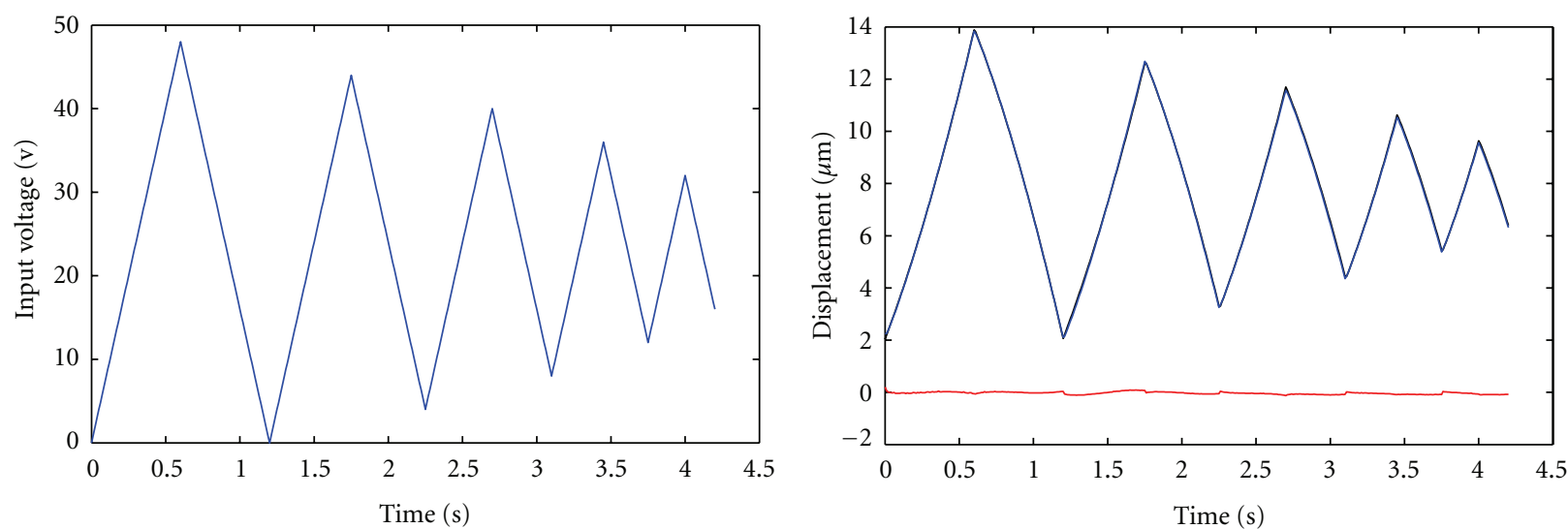

- Model response

— Experimental response

— Modeling error

(a)
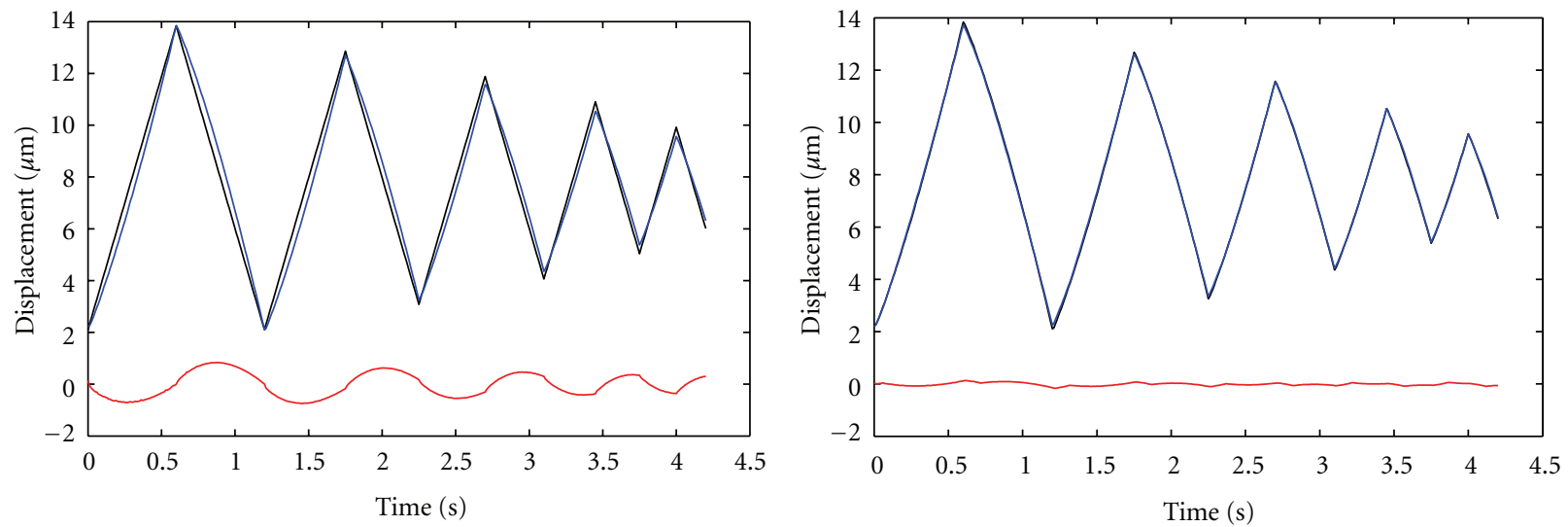

—_ Model response
Experimental response
_ Modeling error

- Model response

— Experimental response

— Modeling error

(c)

(d)

FIgURE 3: (a) The first-order reversal signal. (b) The experimental response and the proposed model response. (c) The experimental response and the linear model response, and (d) the experimental response and the PI model response.

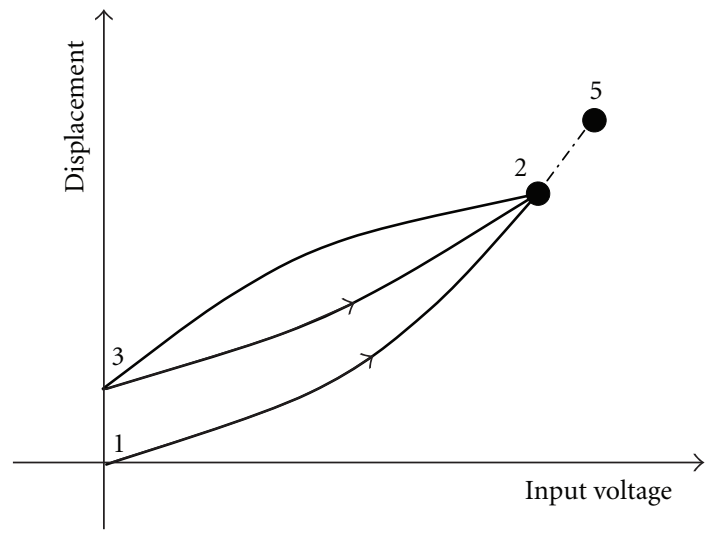

Figure 4: Hysteresis curve movement path. 


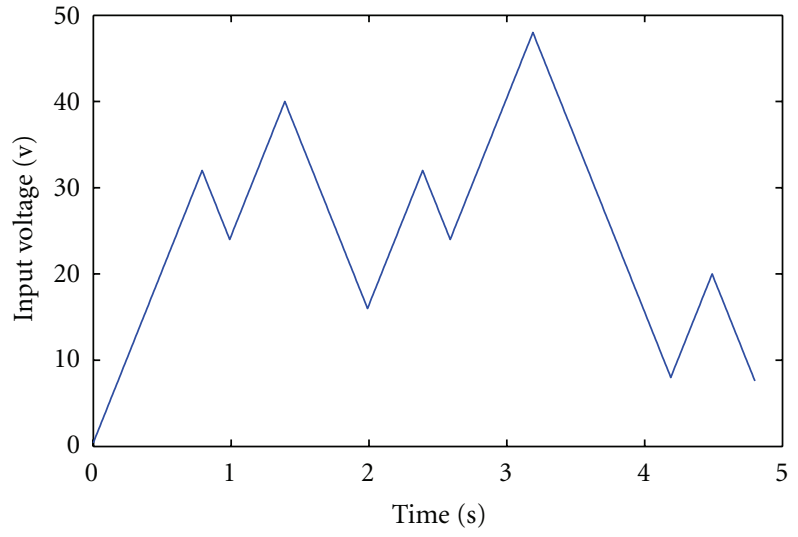

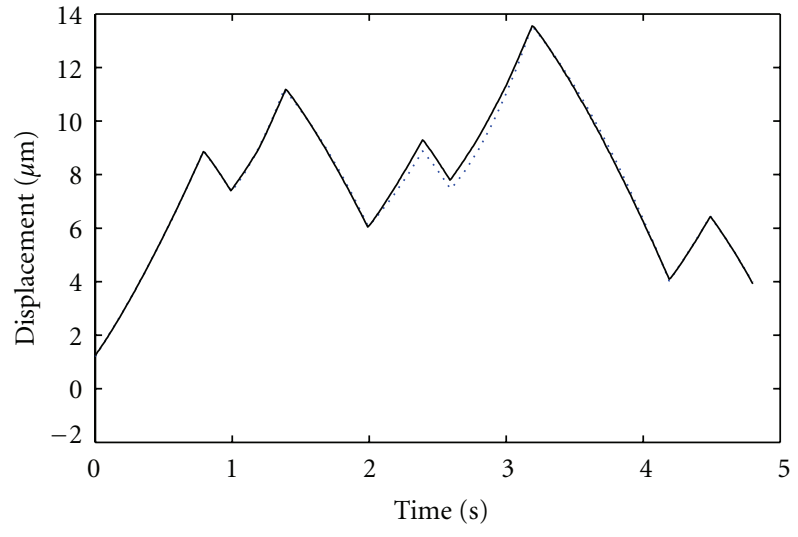

Model response

_ Experimental response

(b)

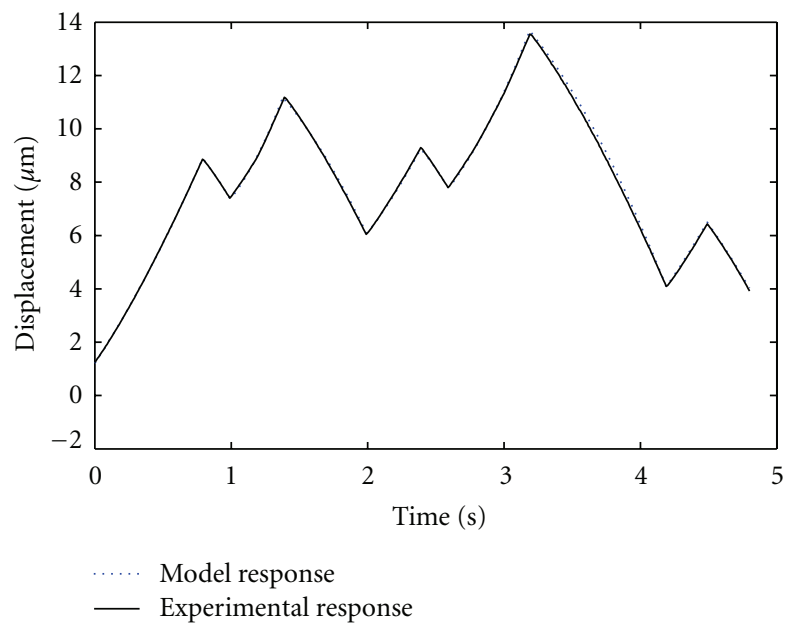

(c)

Figure 5: (a) The experimental input signal. (b) The experimental response and the proposed model response, and (c) the experimental response and the saturated memory model response.

\section{Conclusions}

Hysteresis is the main nonlinearity in piezoelectric actuators. Based on the similarities of the hysteresis trajectory, a new mathematical hysteresis model was proposed to capture the hysteresis behavior in this paper. Utilizing the mathematical transformation, a memory-based modeling framework was developed and experimentally validated on a piezoelectric actuator. Moreover, a new converging point was updated to compensate the prediction error when the hysteresis path hits the upper converging point. The experimental and simulation results demonstrate the effectiveness of the saturated memory model.

\section{Acknowledgments}

This work is supported by the National Natural Science Foundation of China (61174044) and the Shandong Province Natural Science Foundation (ZR2010FM016).

\section{References}

[1] S. Devasia, E. Eleftheriou, and S. O. R. Moheimani, "A survey of control issues in nanopositioning," IEEE Transactions on Control Systems Technology, vol. 15, no. 5, pp. 802-823, 2007.

[2] A. A. Adly, I. D. Mayergoyz, and A. Bergqvist, "Preisach modeling of magnetostrictive hysteresis," Journal of Applied Physics, vol. 69, no. 8, pp. 5777-5779, 1991.

[3] D. Croft, G. Shed, and S. Devasia, "Creep, hysteresis, and vibration compensation for piezoactuators: atomic force microscopy application," Journal of Dynamic Systems, Measurement and Control, Transactions of the ASME, vol. 123, no. 1, pp. 35-43, 2001.

[4] C. Natale, F. Velardi, and C. Visone, "Identification and compensation of Preisach hysteresis models for magnetostrictive actuators," Physica B, vol. 306, no. 1-4, pp. 161-165, 2001.

[5] P. Ge and M. Jouaneh, "Modeling hysteresis in piezoceramic actuators," Precision Engineering, vol. 17, no. 3, pp. 211-221, 1995.

[6] P. Ge and M. Jouaneh, "Tracking control of a piezoceramic actuator," IEEE Transactions on Control Systems Technology, vol. 4, no. 3, pp. 209-216, 1996. 
[7] P. Ge and M. Jouaneh, "Generalized preisach model for hysteresis nonlinearity of piezoceramic actuators," Precision Engineering, vol. 20, no. 2, pp. 99-111, 1997.

[8] X. Tan and J. S. Baras, "Modeling and control of hysteresis in magnetostrictive actuators," Automatica, vol. 40, no. 9, pp. 1469-1480, 2004.

[9] X. Tan, J. S. Baras, and P. S. Krishnaprasad, "Control of hysteresis in smart actuators with application to micro-positioning," Systems and Control Letters, vol. 54, no. 5, pp. 483-492, 2005.

[10] X. Tan and J. S. Baras, "Adaptive identification and control of hysteresis in smart materials," IEEE Transactions on Automatic Control, vol. 50, no. 6, pp. 827-839, 2005.

[11] G. Song, J. Zhao, X. Zhou, and J. A. De Abreu-García, “Tracking control of a piezoceramic actuator with hysteresis compensation using inverse Preisach model," IEEE/ASME Transactions on Mechatronics, vol. 10, no. 2, pp. 198-209, 2005.

[12] K. Kuhnen and H. Janocha, "Adaptive inverse control of piezoelectric actuators with hysteresis operators," in Proceedings of the European Control Conference, Karsruhe, Germany, 1999, paper F 0291.

[13] P. Krejci and K. Kuhnen, "Inverse control of systems with hysteresis and creep," IEE Proceedings, vol. 148, no. 3, pp. 185192, 2001.

[14] S. Bashash and N. Jalili, "Robust multiple frequency trajectory tracking control of piezoelectrically driven micro/nanopositioning systems," IEEE Transactions on Control Systems Technology, vol. 15, no. 5, pp. 867-878, 2007.

[15] H. Jiang, H. Ji, J. Qiu, and Y. Chen, "A modified prandtlishlinskii model for modeling asymmetric hysteresis of piezoelectric actuators," IEEE Transactions on Ultrasonics, Ferroelectrics, and Frequency Control, vol. 57, no. 5, pp. 1200-1210, 2010.

[16] M. Al Janaideh, Y. Feng, S. Rakheja, C. Y. Su, and C. A. Rabbath, "Hysteresis compensation for smart actuators using inverse generalized prandtl-ishlinskii model," in 2009 American Control Conference, ACC 2009, pp. 307-312, usa, June 2009.

[17] M. Al Janaideh, S. Rakheja, and C. Y. Su, "An analytical generalized Prandtl-Ishlinskii model inversion for hysteresis compensation in micropositioning control," IEEE/ASME Transactions on Mechatronics, vol. 16, no. 4, pp. 734-744, 2011.

[18] Q. Xu and P.-K. Wong, "Hysteresis modeling and compensation of a piezostage using least squares support vector machines," Mechatronics, vol. 21, no. 7, pp. 1239-1251, 2011.

[19] P.-K. Wong, Q. Xu, C.-M. Vong, and H.-C. Wong, "Ratedependent hysteresis modeling and control of a piezostage using online support vector machine and relevance vector machine," IEEE Transactions on Industrial Electronics, vol. 59, no. 4, pp. 1988-2001, 2012.

[20] J.-J. Tzen, S. L. Jeng, and W. H. Chieng, "Modeling of piezoelectric actuator for compensation and controller design," Precision Engineering, vol. 27, no. 1, pp. 70-86, 2003.

[21] L. Sun, C. Ru, W. Rong, L. Chen, and M. Kong, "Tracking control of piezoelectric actuator based on a new mathematical model," Journal of Micromechanics and Microengineering, vol. 14, no. 11, pp. 1439-1444, 2004.

[22] S. Bashash and N. Jalili, "Underlying memory-dominant nature of hysteresis in piezoelectric materials," Journal of Applied Physics, vol. 100, no. 1, Article ID 014103, pp. 1-6, 2006.

[23] S. Bashash and N. Jalili, "A polynomial-based linear mapping strategy for feedforward compensation of hysteresis in piezoelectric actuators," Journal of Dynamic Systems, Measurement and Control, vol. 130, no. 3, Article ID 031008, pp. 1-10, 2008.
[24] S. Bashash, N. Jalili, P. Evans, and M. J. Dapino, "Recursive memory-based hysteresis modeling for solid-state smart actuators," Journal of Intelligent Material Systems and Structures, vol. 20, no. 18, pp. 2161-2171, 2009. 

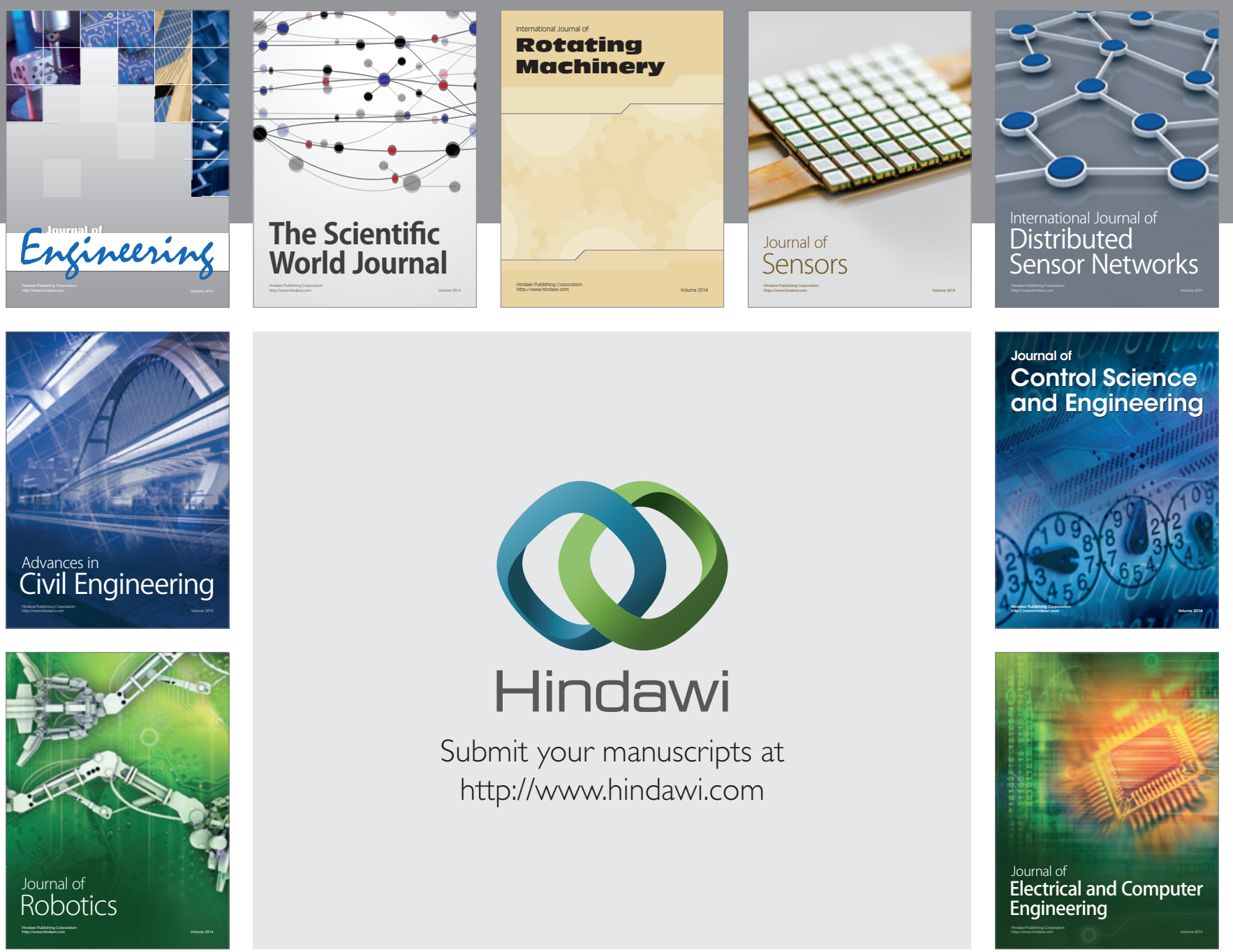

Submit your manuscripts at

http://www.hindawi.com
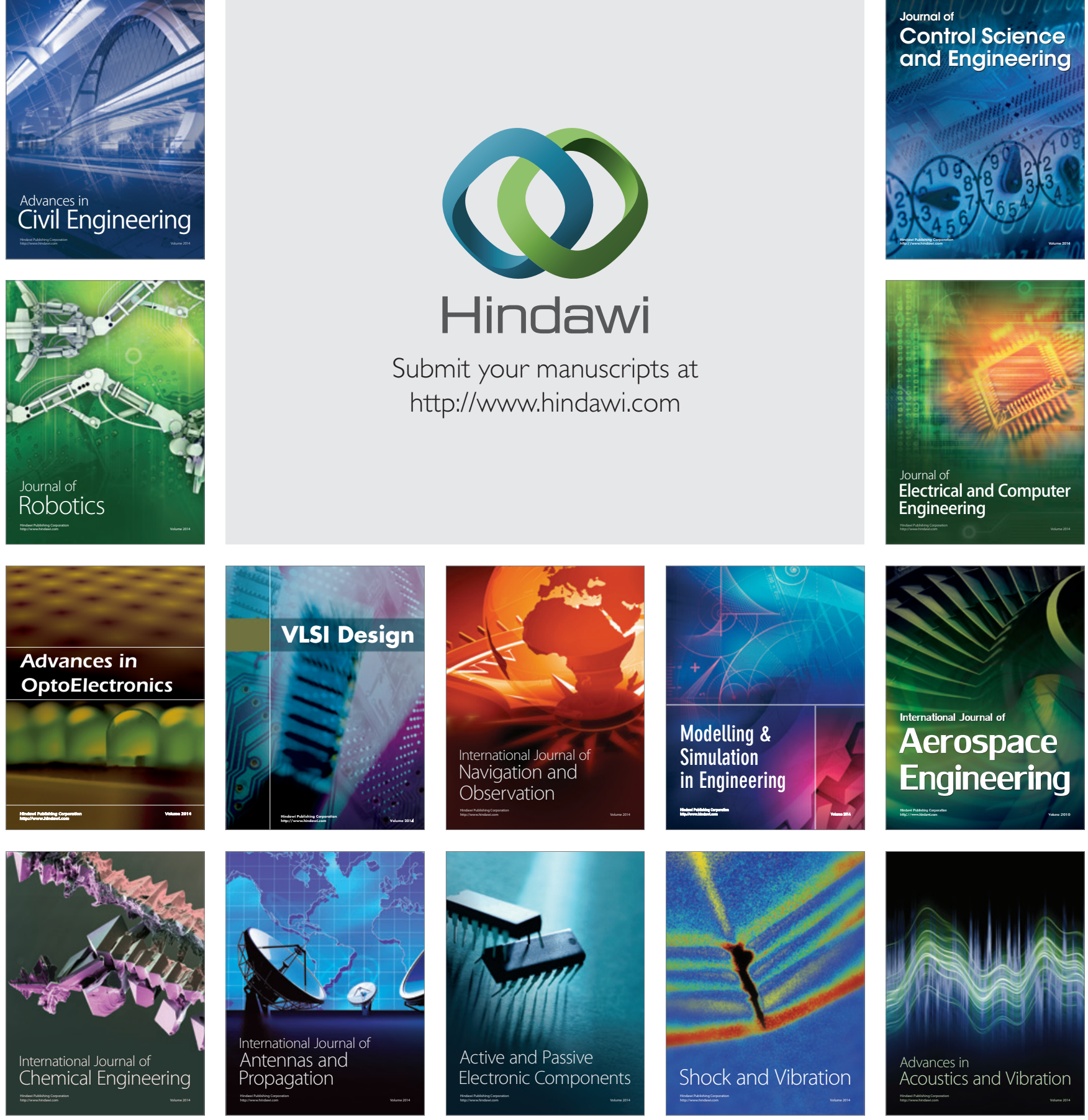